\title{
Reentrance Phenomenon of a mixed spin Ising model
}

\author{
Hadey K. Mohamad \\ Department of Physics, College of Science, Al-Muthanna University, Samawa550, Iraq. \\ E-mail address: hadeyk2002@yahoo.com
}

Keywords: Mixed-spin Ising model; Single-ion anisotropy; Reentrant behaviour.

\begin{abstract}
A reentrant magnetic phase transition in molecular based magnet $\mathrm{AFe}^{\mathrm{II}} \mathrm{Fe}^{\mathrm{III}}\left(\mathrm{C}_{2} \mathrm{O}_{4}\right)_{3}\left[\mathrm{~A}=\mathrm{N}\left(n-\mathrm{C}_{n} \mathrm{H}_{2 n+1}\right)_{4}, n=3-5\right]$, is investigated by the use of Mean-Field Theory(MFT) for a mixed spin- 2 and spin-5/2 Ising system on a simple cubic lattice. It is found that a double-entrant ferrimagnetic behaviour in the mixed-spin system depends on the negative values of the anisotropies in the order-disorder system for the two sublattices of the mixture. It is shown using numerical calculations that this phenomenon is strongly affected by the magnetic anisotropy and lattice disorder.
\end{abstract}

\section{INTRODUCTION}

Recently, a lot of efforts has been directed to the ferrimagnetic materials, specially due to their great potential for technological applications [1]. Ferrimagnets are materials where ions on different sublattices have opposing magnetic moments and show paramagnetic behaviour above transition temperature $\left(T_{c}\right)$. J.Oitmaa et al introduced a mixed-spin Ising model on the simple cubic lattice which showed reentrant behaviours [2]. Other researchers in this respect observed that the effect of next-nearest-neighbour interaction between the spin sites on the lattice possibly leads to a reentrant transition as well the single-ion anisotropy may cause a reentrant one $[3,4,5]$. The reentrant behaviour does occur in the compounds having composition close to $A_{0.5} A_{0.5}^{\prime} \mathrm{MnO}_{3}$ (where $A=L a, \operatorname{Pr}, N d \ldots .$. rare metals; $A^{\prime}=S r, B a, C a \ldots \ldots$ ) [6]. It is worth to note that the origin of reentrant phenomenon is competition between two opposites: ferromagnetic and antiferromagnetic interactions present in materials. Here, it has been studied a mixed-spin Ising ferrimagnetic system on a simple cubic lattice in which the two mixing sublattices have spins two $(0, \pm 1, \pm 2)$ and spins five-half( $\pm 1 / 2, \pm 3 / 2, \pm 5 / 2)$. In this work we have studied, within a mean-field approximation, the reentrant phenomenon of the model. We found that the system considered exhibits a double entrant behaviour at low temperatures.

\section{THE MODEL}

It has been studied a 3 -D Ising model with spin $\mu_{i}^{A}=0, \pm 1, \pm 2$ and $\mu_{j}^{B}= \pm 1 / 2, \pm 3 / 2, \pm 5 / 2$, located in alternating sites of a simple cubic lattice, such that the $\mu_{i}$ and $\mu_{j}$ are the nearest neighbours. A Hamiltonian for this model includes only first nearest neighbour, with $\mathrm{H}=0$, that :

$$
H=-J \sum_{i, j} \mu_{i}^{A} \mu_{j}^{B}-D_{A} \sum_{i}\left(\mu_{i}^{A}\right)^{2}-D_{B} \sum_{j}\left(\mu_{j}{ }^{B}\right)^{2}
$$

where $D_{A}, D_{B}$ are the anisotropies acting on the spins $\mu_{i}$ and $\mu_{j}$ respectively. $J$ is the nearest neighbour exchange parameter.

Within the framework of the mean-field method, it is necessary to evaluate the following expectation values for the considered spin problems in this work, that :

$$
m_{A} \equiv\left\langle\mu_{i}^{A}\right\rangle_{o}=\frac{e^{-3 \beta D_{A}} \sinh \left(\beta z J m_{B}\right)+2 \sinh \left(2 \beta z J m_{B}\right)}{e^{-3 \beta D_{A}} \cosh \left(\beta z J m_{B}\right)+\cosh \left(2 \beta z J m_{B}\right)+0.5 e^{-4 \beta D_{A}}}
$$




$$
m_{B} \equiv\left\langle\mu_{j}^{B}\right\rangle_{o}=\frac{1}{2} \frac{5 \sinh \left(\frac{5}{2} \beta z J m_{A}\right)+3 e^{-4 \beta D_{B}} \sinh \left(\frac{3}{2} \beta z J m_{A}\right)+e^{-6 \beta D_{B}} \sinh \left(\frac{1}{2} \beta z J m_{A}\right)}{\cosh \left(\frac{5}{2} \beta z J m_{A}\right)+e^{-4 \beta D_{B}} \cosh \left(\frac{3}{2} \beta z J m_{A}\right)+e^{-6 \beta D_{B}} \cosh \left(\frac{1}{2} \beta z J m_{A}\right)}
$$

The free energy of the system is obtained from a mean field calculation of the system Hamiltonian based on the Bogoliubov inequality $[5,7,8,9]$ :

$$
F \leq F_{0}+\left\langle H-H_{0}\right\rangle_{0}
$$

where $F$ is the free energy of $H$ given by relation (1), that

$$
F=-k_{B} T \ln Z ; Z=\sum_{i, j} e^{-\beta H}
$$

$F_{0}$ is the free energy of a paramagnetic phase and $H_{0}$ a trial Hamiltonian depending on variational parameters, that

$$
F_{0}=-k_{B} T \ln Z_{0} ; Z_{0}=\sum_{i, j} e^{-\beta H_{0}}
$$

$\mathrm{Z}, Z_{0}$ are the true partition function and trial one respectively.

In this research we consider one of the possible choices of $H_{0}$, namely:

$$
H_{0}=-\sum_{i}\left[\lambda_{A} \mu_{i}^{A}+D_{A}\left(\mu_{i}^{A}\right)^{2}\right]-\sum_{j}\left[\lambda_{B} \mu_{j}^{B}+D_{B}\left(\mu_{j}^{B}\right)^{2}\right]
$$

where $\lambda_{A}$ and $\lambda_{B}$ are the two variational parameters related to the two different spins $\mu_{i}{ }^{A}$ and $\mu_{j}^{B}$ respectively.

The approximated free energy of the system considered is obtained by minimizing the right side of equation (4) with respect to variational parameters. Thus, we can evaluate the expression in eq.(4), that :

$$
\begin{aligned}
& f=-\frac{1}{2 \beta}\left\{\operatorname { l n } \left[2 e^{1 / 4 \beta D_{A}} \cosh \left(\frac{1}{2} \beta z J m_{B}\right)+2 e^{9 / 4 \beta D_{A}} \cosh \left(\frac{3}{2} \beta z J m_{B}\right)\right.\right. \\
& \left.\left.+2 e^{25 / 4 \beta D_{A}} \cosh \left(\frac{5}{2} \beta z J m_{B}\right)\right]+\ln \left[2 e^{\beta D_{B}} \cosh \left(\beta z J m_{A}\right)+2 e^{4 \beta D_{B}} \cosh \left(2 \beta z J m_{A}\right)+1\right]\right\} \\
& +1 / 2 z J m_{A} m_{B}
\end{aligned}
$$

where $\beta=\frac{1}{K_{B} T}$, and $\mathrm{z}(\mathrm{z}=6)$ its nearest-neighbor coordination number of a simple cubic lattice. 


\section{RESULTS AND DISCUSSIONS}

First, it has been evaluated the approximated magnetic moments of a mixed spin-2 and spin-5/2 on a simple cubic lattice with different single-ion anisotropies. The numerical results are presented in Figs.((1),(2)), selecting typical values of $D_{A} /|J|$ for fixed values of $D_{B} /|J|$.

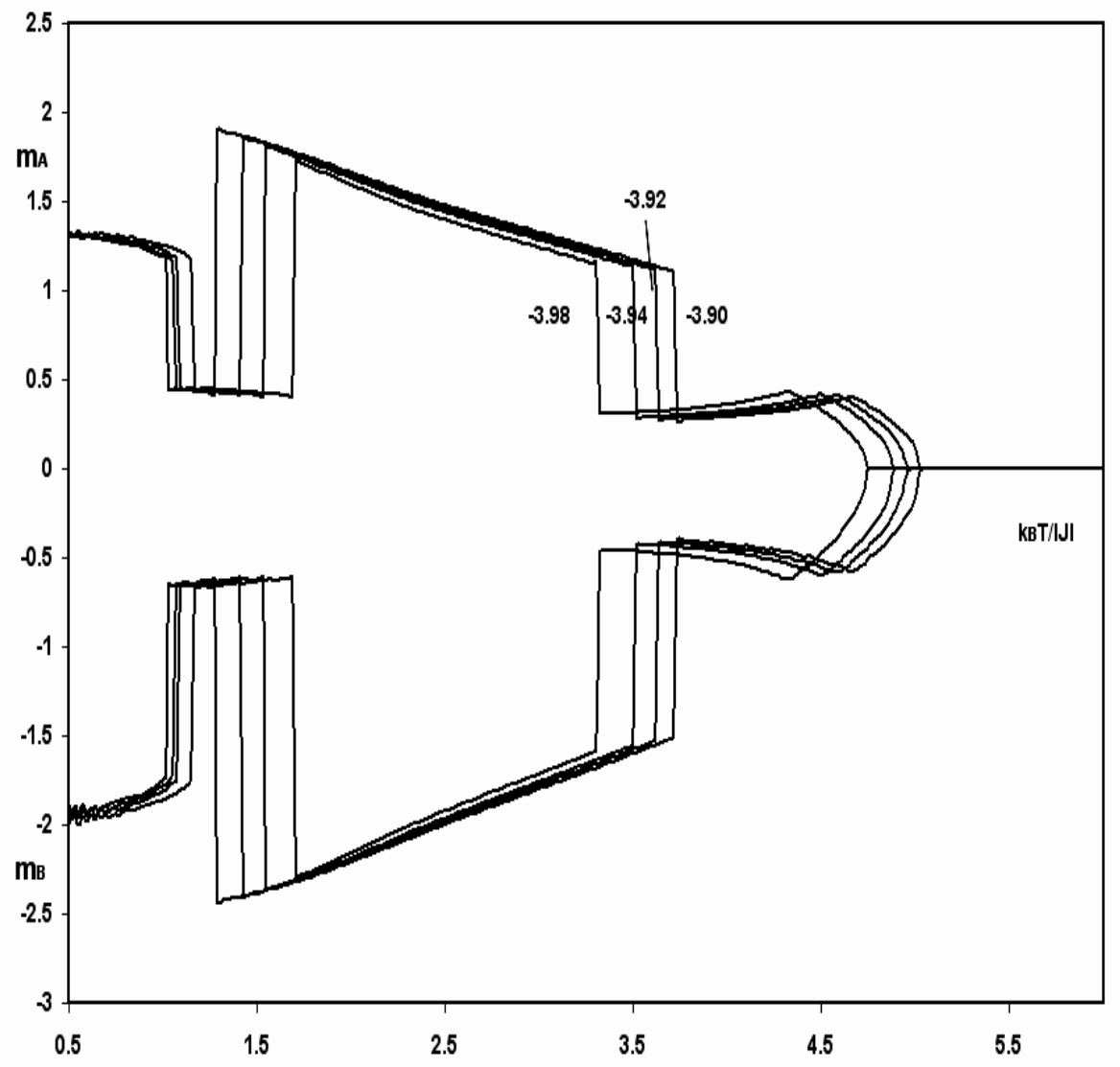

Fig.1. Thermal variations of the sublattice magnetization $m_{A}, m_{B}$ for the mixed -spin Ising ferrimagnet with the coordination number $z=6$, when the value of $D_{A} /|J|$ is changed, for fixed $D_{B} /|J|=-2.0$. The curves with values of $D_{A} / J \mid=-3.90,-3.98$ indicate a double-entrant behavior at low temperature. 


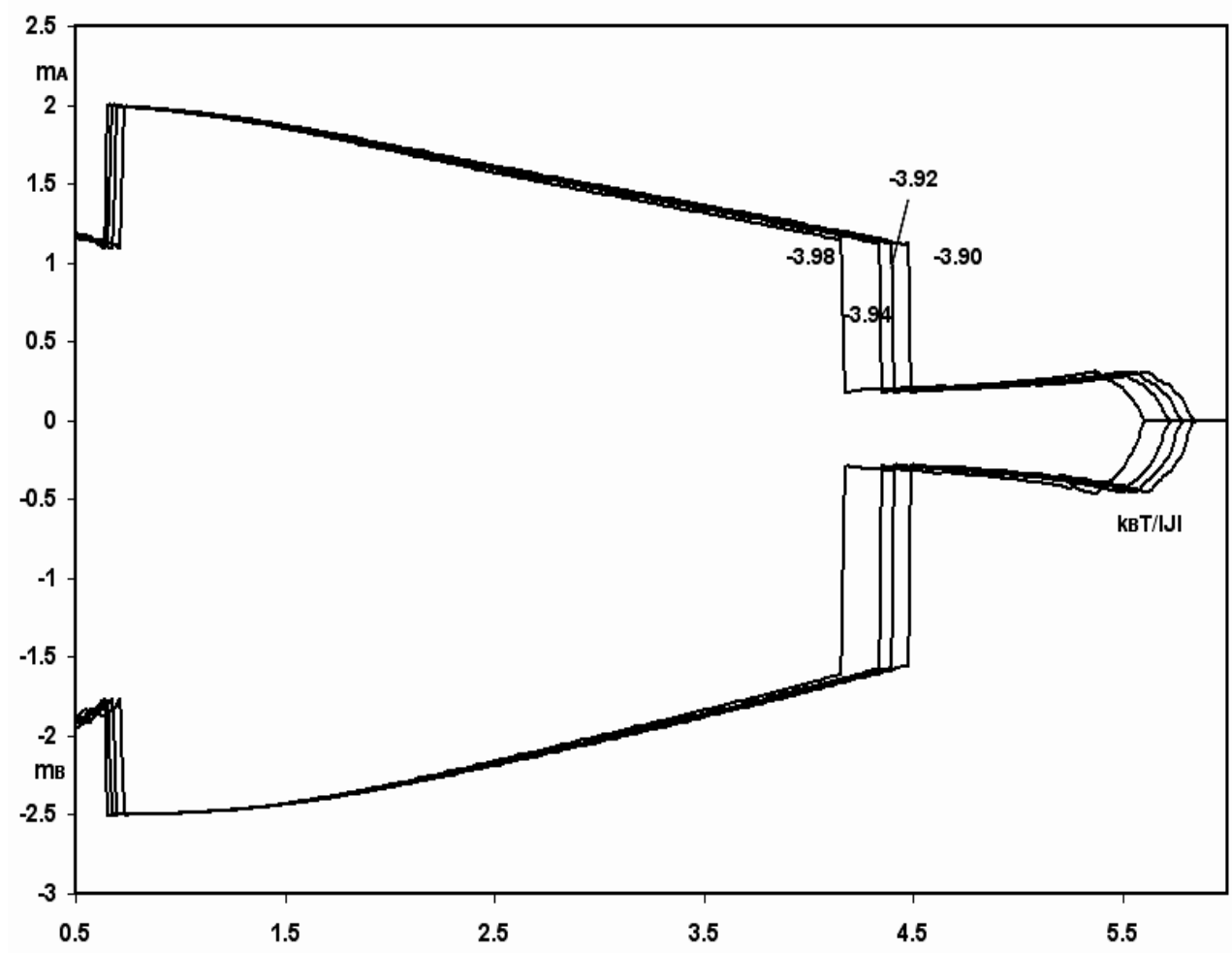

Fig.2. Thermal variations of the sublattice magnetization $m_{A}, m_{B}$ for the mixed-spin Ising ferrimagnet with the coordination number $\mathrm{z}=6$, when the value of $D_{A} /|J|$ is changed, for fixed $D_{B} /|J|=-1.8$. The curves with values of $D_{A} /|J|=-3.90,-3.98$ indicate a reentrant behavior at low temperature.

The sublattice magnetization curves labeled from -3.98 to -3.90 are for the values of $D_{A} /|J|$ corresponding to the critical points. Fig.(1) and Fig.(2) show the sublattice magnetization curves at low temperature for anisotropy $D_{A} /|J|$ corresponding to the reentrant behaviour as in Fig.(3). 


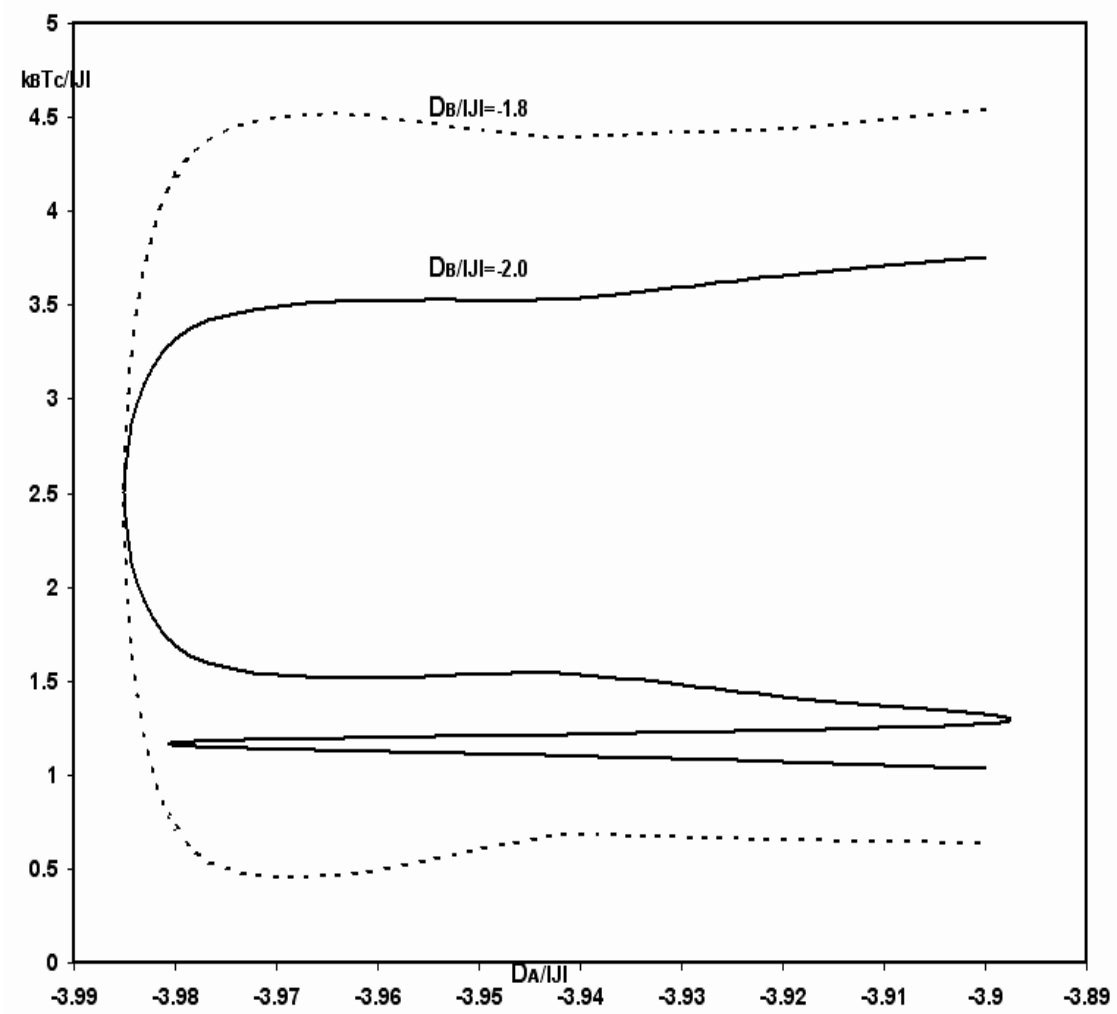

Fig.3. Low-temperature phase diagrams in the $\left(D_{A}, T\right)$ plane for the mixed-spin Ising ferrimagnet with the coordination number $\mathrm{z}=6$, in the region of $D_{A}$ at $D_{B} /|J|=-1.8,-2.0$, respectively.

Now we may discuss the scale of Fig.(3) in which the critical lines for $D_{B} /|J|=-2.0, D_{B} /|J|=-1.8$ show double-entrant behaviour in phase diagram(three critical temperatures) and reentrant one in phase diagram(two critical temperatures), respectively. These results are consistent with predictions derived from Figs.((1),(2)). At very low temperature the system will be disordered, since $D_{A}<0$ favours that $S=0$ at A sites. As the temperature increases the system orders at a lower critical temperature, moves to an ordered phase passing through another two critical points or another critical one near $D_{A} /|J|=-3.99$ when $D_{B} /|J|=-2.0,-1.8$, respectively, then disordered again at the transition temperature $\left(T_{C}\right)[2,3,5]$. Note that such a reentrant behaviour with three consecutive critical points has not been reported in the analogous mixed-spin system on the honeycomb lattice[10]. These transitions of this kind found in the exact treatment of a mixed spin-1/2 and spin-1 Ising model on the diced lattice[3]. It is noteworthy that the results obtained can provide the analytical background for further investigations on the effects of crystal field and boundary conditions on the critical properties of the Ising model.

\section{CONCLUSION}

We have applied MFA to the study of a mixed spin-2 and spin-5/2 Ising model on a simple cubic lattice modeled by Eq.(1) to examine a characteristic feature of $A \mathrm{Fe}^{\mathrm{II}} \mathrm{Fe}^{\mathrm{III}}\left(\mathrm{C}_{2} \mathrm{O}_{4}\right)_{3}\left[A=N\left(n-C_{n} H_{2 n+1}\right)_{4}, n=3-5\right]$. In particular, we have studied the effect of a single-ion anisotropy on the existence of the reentrant ferrimagnetism phenomenon. We have shown that the system undergoes a double reentrant behaviour in the phase diagrams as shown in Fig.(1).It is also interesting to remark that the first-order transition lines always exhibit a reentrant 
behaviour at low temperature as shown in Fig.(2), i.e., two phase transitions[2,5]. These behaviors started from the disordered ground state before entering the ordered phase at lower critical temperature. Thus the ferrimagnetic long-range order disappears due to the thermal fluctuations at the upper critical temperature $T_{C}$. We hope that this work could stimulate experimental and theoretical works on the system which is considered.

\section{References}

[1] W.Figueiredo, M.Godoy, V.S.Leite, Braz.J.Phys.,34(2004)392.

[2] J.Oitmaa, W.Zheng, phys.A,328(2003)185.

[3] M.Jascur,J.Strecka, Cond.Matt.phys,8(2005)869.

[4] M.Zukovic, A.Bobak,J.Magn.Magn.Mater.,170(1997)49.

[5] O.F.Abubrig,D.Horvath,A.Bobak,M.Jascur,Physica A,296,(2001)437.

[6] B.T.Cong,P.N.A.Huy,N.H.Long,D.D.Long,Bull.Mter.Sci.,26,1(2003)151.

[7] A.Benyoussef,A.ElKenz,M.Elyadari,M.J.Condensed Matter,8,1(2007)72.

[8] HadeyK.Mohamad,E.P.Domashevskaya,A.F.Klinskikh,Phys.A,388(2009)4713.

[9] Hadey K. Mohamad, J. Int. Acad. Res. Multi. 2(2014)342.

[10]T.Kaneyoshi, Y.Nakamura, S.Shin,J.phys.:Condens.Matter,10(1998)7025. 